University of New Hampshire

University of New Hampshire Scholars' Repository

Space Science Center

Institute for the Study of Earth, Oceans, and

Space (EOS)

8-27-2010

\title{
Simulations of a monolithic lanthanum bromide gamma-ray detector
}

\author{
Camden Ertley \\ University of New Hampshire - Main Campus \\ Chris Bancroft \\ University of New Hampshire \\ Peter F. Bloser \\ University of New Hampshire, Peter.Bloser@unh.edu \\ Taylor Connor \\ University of New Hampshire - Manchester \\ Jason S. Legere \\ University of New Hampshire, jslegere@unh.edu
}

See next page for additional authors

Follow this and additional works at: https://scholars.unh.edu/ssc

Part of the Astrophysics and Astronomy Commons

\section{Recommended Citation}

Camden Ertley ; Christopher Bancroft ; Peter Bloser ; Taylor Connor ; Jason Legere ; Mark McConnell and James Ryan "Simulations of a monolithic lanthanum bromide gamma-ray detector", Proc. SPIE 7805, Hard X-Ray, Gamma-Ray, and Neutron Detector Physics XII, 78051X (August 27, 2010); doi:10.1117/12.859725; http://dx.doi.org/10.1117/12.859725

This Conference Proceeding is brought to you for free and open access by the Institute for the Study of Earth, Oceans, and Space (EOS) at University of New Hampshire Scholars' Repository. It has been accepted for inclusion in Space Science Center by an authorized administrator of University of New Hampshire Scholars' Repository. For more information, please contact Scholarly.Communication@unh.edu. 


\section{Authors}

Camden Ertley, Chris Bancroft, Peter F. Bloser, Taylor Connor, Jason S. Legere, Mark L. McConnell, and James M. Ryan 


\title{
Simulations of a monolithic lanthanum bromide gamma-ray detector
}

\author{
Camden Ertley, Christopher Bancroft, Peter Bloser, Taylor Connor, \\ Jason Legere, Mark McConnell, and James Ryan \\ Space Science Center, Univ. of New Hampshire, Durham, NH USA 03824
}

\begin{abstract}
We have been working on the development of a detector design for a large area coded aperture imaging system operating in the $10-600 \mathrm{keV}$ energy range. The detector design is based on an array of Lanthanum Bromide $\left(\mathrm{LaBr}_{3}\right)$ scintillators, each directly coupled to a Hamamatsu 64-channel multi-anode photomultiplier tube (MAPMT). This paper focuses on the development of the GEANT4-based simulations as an aid in the optimization of the detector design. The simulations have been validated by comparisons with various laboratory data sets. We will summarize the current status and latest findings from this study.
\end{abstract}

Keywords: Gamma-Ray Astronomy, Scintillators, Monte Carlo Simulations

\section{INTRODUCTION}

In 2003 NASA announced the Beyond Einstein program to use as a roadmap for the Structure and Evolution of the Universe theme ${ }^{1}$. As part of this program, the Black Hole Finder Probe (BHFP) mission would perform an all sky census of accreting black holes. The goal was to study black hole formation and evolution. The Coded Aperture Survey Telescope for Energetic Radiation (CASTER) ${ }^{2}$, one of two proposed concepts for the BHFP, is based on the Anger camera principal $^{3}$. To achieve the goals of BHFP, CASTER would need a large field of view, $60^{\circ} \times 120^{\circ}$, and cover the $10-600 \mathrm{keV}$ energy range ${ }^{4}$. The large field of view would allow CASTER to scan a large portion of the sky during each orbit. The upper limit to this energy range was chosen in order to cover the $511 \mathrm{keV}$ line, allowing CASTER to be sensitive to high energy processes including electron positron annihilation. An array of Anger camera modules consisting of a coded mask positioned above an imaging detector plane would achieve BHFP goals. Figure 1 shows the proposed Anger camera module. The detector plane is a $12 \times 12$ array of detector modules, see Figure 2. An individual detector module would be a $\mathrm{LaBr}_{3}$ scintillation crystal directly coupled to a multi-anode photomultiplier tube (MAPMT) to provide readout of the light distribution within the crystal, thus providing event location information. $\mathrm{LaBr}_{3}$ was chosen because of its high light output $(63000$ per $\mathrm{MeV})$, fast decay times $(0.016 \mu \mathrm{s})$, and its excellent energy resolution $(6.6 \% \text { at } 122 \mathrm{keV})^{5}$.

Though the future status of the Beyond Einstein program is very uncertain, continuing research on the proposed CASTER detector is still important. In addition to its application in astrophysics, many other fields, such as nuclear medicine, could benefit by advances in this type of detector technology.

The CASTER detector design should be optimized for both energy and position resolution in the $10-600 \mathrm{keV}$ energy range. To facilitate the optimization of the design, a Monte Carlo simulation has been created using the Geant 4 toolkit ${ }^{6}$. This paper focuses on the validation of the simulations using experimental data collected using a laboratory ${ }^{57} \mathrm{Co}$ gamma ray sources.

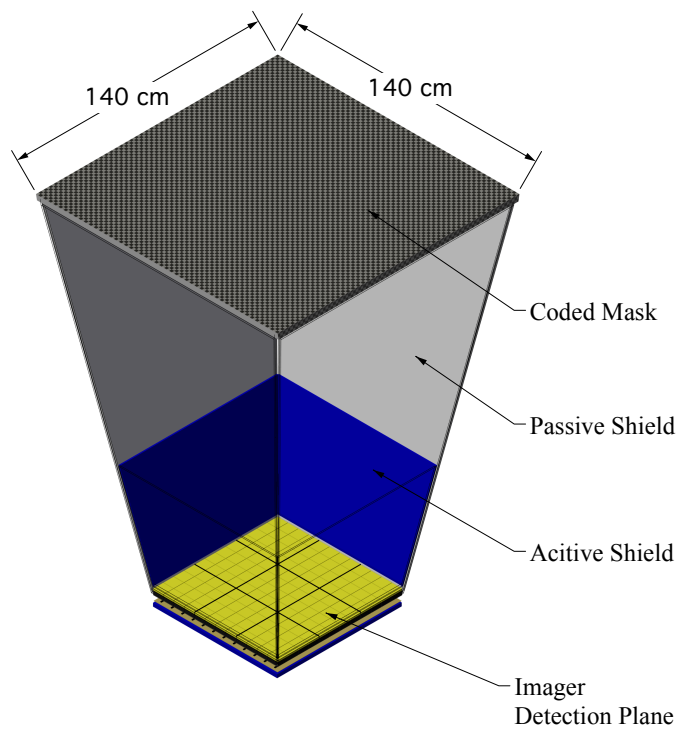

Figure 1: CASTER would consist of an array of Anger camera modules. Each module is made from a coded mask positioned above an imager detection plane. 


\section{PROTOTYPE DETECTOR}

A prototype of a CASTER detector module has been fabricated for laboratory testing. The $\mathrm{LaBr}_{3}$ crystal is a $50 \mathrm{~mm} \times 50 \mathrm{~mm} \times 10 \mathrm{~mm}$ Saint Gobain Crystals Brillance $380^{7}$. Because of the hygroscopic nature of the $\mathrm{LaBr}_{3}$ crystal, it must be contained within a hermetically sealed enclosure. To avoid an extra layer of glass between the crystal and the MAPMT, Saint Gobain provided a custom assembly that optically coupled a 64-channel Hamamatsu H8500 MAPMT ${ }^{8}$ directly to the crystal, all hermetically contained within a single housing. The $\mathrm{LaBr}_{3}$ has surface treatments to increase the reflectivity on the top (front) surface and to increase photon absorption on the side surfaces. These surface properties are routinely used to provide good position resolution in medical gamma cameras. The top and side surfaces were roughened to prevent light loss while the bottom surface connected to the PMT was polished to facilitate light transmission to the photocathode. A $0.3 \mathrm{~mm}$ sheet of Teflon covers the top surface to further increase the reflectivity of that surface. The sides are wrapped in a black absorber to increase photon absorption.

\section{LABORATORY SETUP}

Collection of data from the prototype detector was required for the validation of the Monte Carlo simulations (Section 4). These data included flood tests to uniformly irradiate the scintillator and also a series of collimated surface scans to determine the detector response as a function of interaction location $(\mathrm{x}, \mathrm{y})$. The locationdependent parameters of interest include the light distribution amongst the 64 anodes, the spatial resolution, and the energy resolution.

A test stand was constructed to facilitate these measurements. The surface scan data required the capability to scan the face of the detector in small increments with a well collimated source. A tungsten collimator provided a beam spot size of $\sim 500 \mu \mathrm{m}$. A surface scan with the beam centered on each anode (with a 6.08 $\mathrm{mm}$ anode pitch) allows the gain variations of the MAPMT to be determined. A finer scan using a much smaller $(1 \mathrm{~mm})$ pitch will allow position finding algorithms to be developed.

The lab test stand uses a computer controlled Velmex X-Y translation table to position the collimated gamma ray source above the detector. Each anode from the MAPMT was connected to one channel of a CAEN N5683 sixteen channel amplifiers and one channel of a CAEN V785 sixteen channel analog to digital converter. The test stand was controlled and data was collected using a custom LabVIEW program. A picture of the lab setup can be seen in Figure 3.

Processing of the lab data included a renormalization of the anode pulse-heights to account for gain differences in the anodes of the MAPMT. The required normalization factors were derived from anode scan data using a collimated beam from a ${ }^{57} \mathrm{Co}$ source centered on each anode. The factors were chosen such that the position of the full-energy peak in each anode corresponded to the

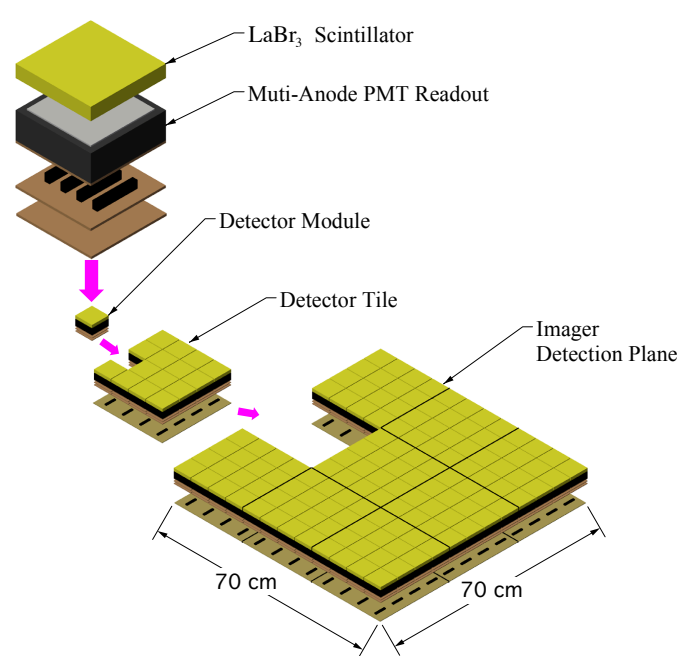

Figure 2: The detector plane in each of the CASTER telescopes consists of a $12 \times 12$ array of detector modules. Each detector module has a $\mathrm{LaBr}_{3}$ scintillator coupled to a multi-anode photomultiplier tube.

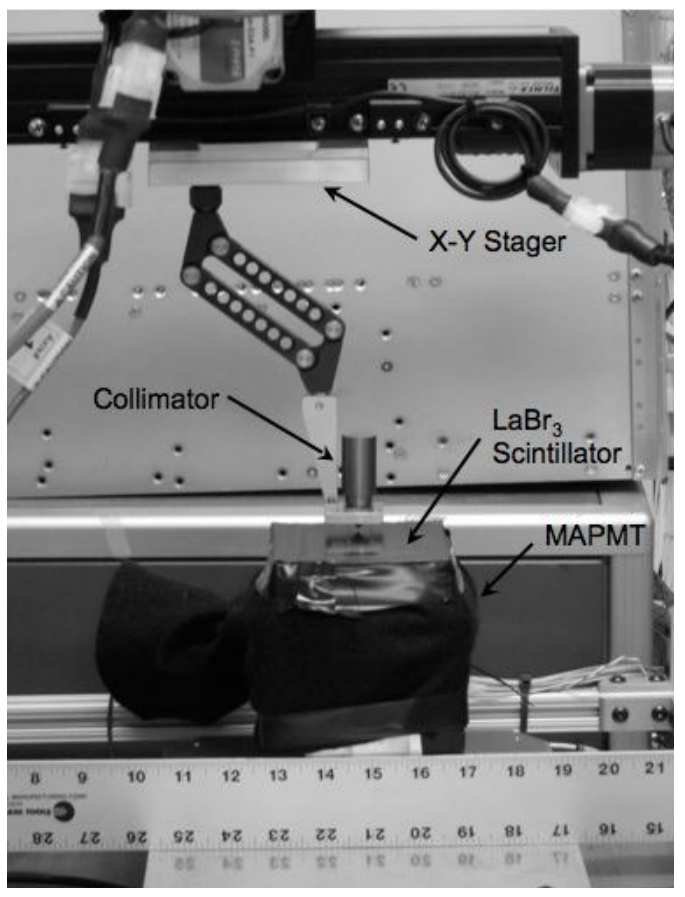

Figure 3: The laboratory setup consists of a collimated gamma ray source positioned above the $\mathrm{LaBr}_{3}$ detector. An X-Y stager is used to position the collimator and source. 
average full-energy peak position of the entire anode array. The normalization factor for a given anode was found by dividing the average full-energy peak position of the entire anode array by the full-energy peak position of that anode. The distribution of the anode normalization factors is shown in Figure 4. The normalization constants have a mean value of 1.018 , with a root mean square of 0.136 .

\section{SIMULATIONS}

The detector design needs to be optimized for position and energy resolution. The position resolution is needed for achieving the fine angular resolution of the coded aperture imager. The energy resolution is needed for spectral studies of astrophysical sources. The scintillator thickness and surface treatments can be varied to optimize these parameters. Instead of building a number of different detectors to optimize the performance, a Monte Carlo simulation has been created using the Geant4 toolkit. Geant4 was chosen because of its ability to easily model radiation sources, different detector configurations, and its accuracy in modeling physical processes. Geant4 has been shown to be a useful tool in the development of scintillator based gamma ray detectors ${ }^{9}$. The simulation includes the laboratory detector assembly (the enclosed scintillator and MAPMT assembly) along with the tungsten collimator and the radiation source (Figure 5).

Geant4 provides several different ways to model boundary optical processes ${ }^{10}$. The GLISUR model was chosen for these simulations because of its ease of implementation. In this model, surface finish properties are defined using one of many possible situations, for example polished, ground, or back painted. A ground surface is made of micro-facets. Each time a photon is reflected, a micro-facet is selected from a distribution and the facet normal is calculated. The facet normals uses a polish parameter, $0 \leq$ polish $\leq 1$, to define the roughness of the material surface. The polish parameter is used to create a vector corresponding to a random point on a sphere of radius $(1-$ polish $)$. The facet normal is the sum of the average surface normal vector and the polish vector. For rough surfaces, polish equal to zero, a Lambertian distribution of the facet normals is assumed. For smooth surfaces, polish equal to one, the possibility of a reflection is calculated using the Fresnel equations. The reflection probability of the surface needs to be defined in the GLISUR model. The reflectivity is defined for each surface with a value of 0 to 1 . Other, more complex, models could be used to model the optical processes. These models use more parameters to calculate the facet normal, which are often difficult to estimate ${ }^{11}$.

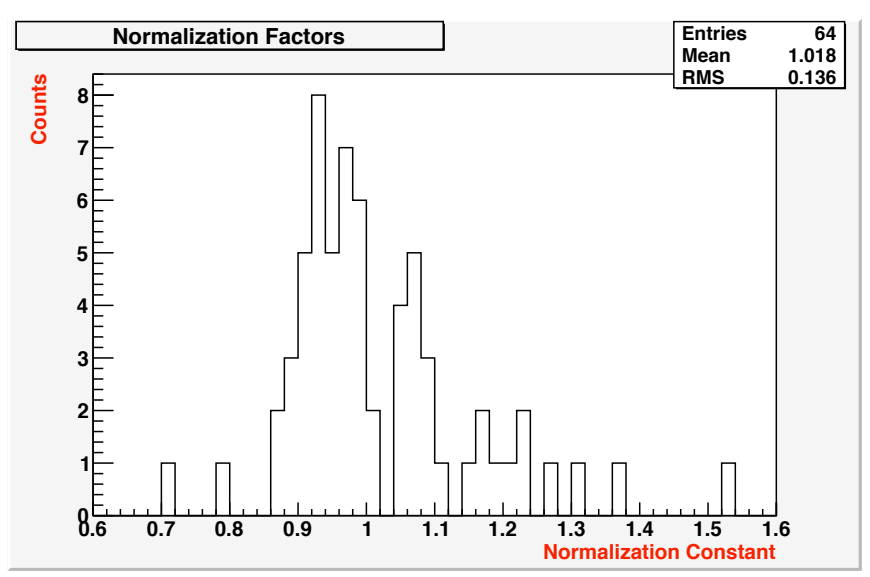

Figure 4: Because of gain variation in the MAPMT, the lab data was normalized. The figure shows a histogram of the normalization constants. The mean is 1.018 and the root mean square is 0.136 .

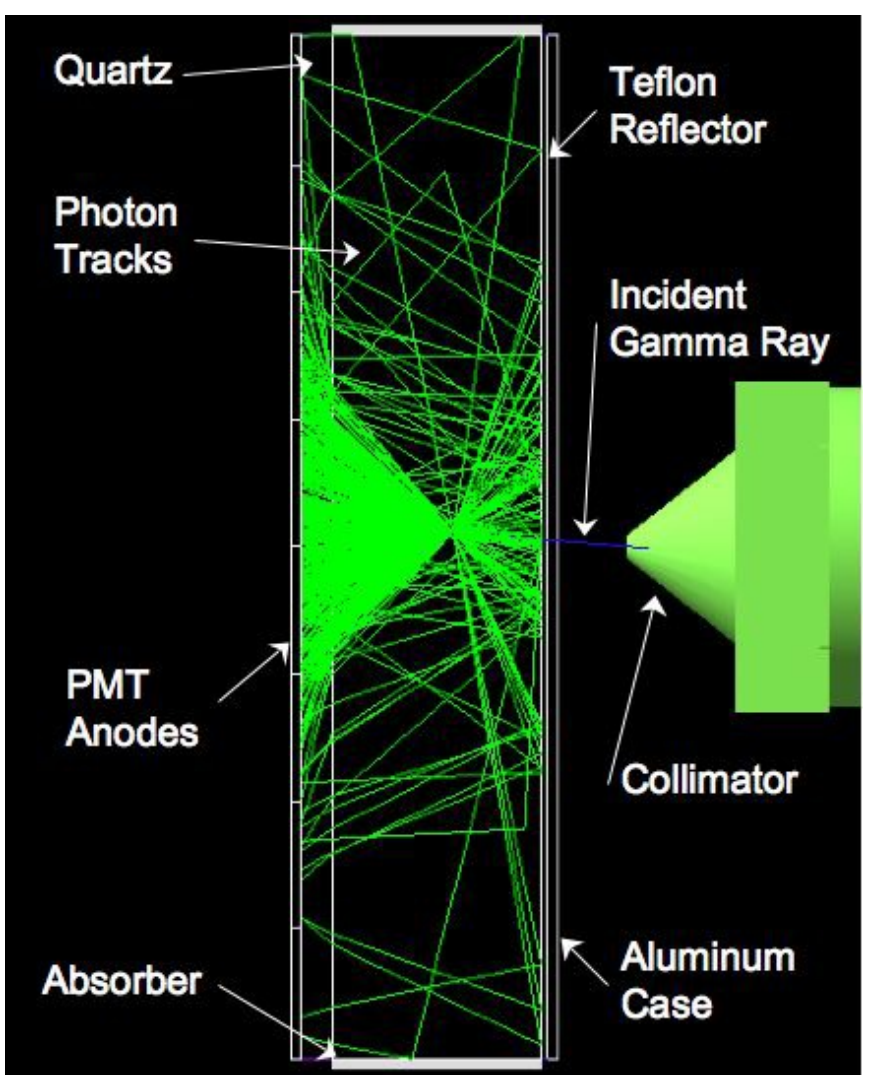

Figure 5: Simulation of a single event in Geant4. An incident gam ma photon interacts in the $\mathrm{LaBr}_{3}$ crystal. The resulting photons are tracked as they propagate through the crystal, glass, and vacuum. When they contact a PMT anode, they photon is recorded and destroyed. Only photons that are recorded by the PMT anodes are 


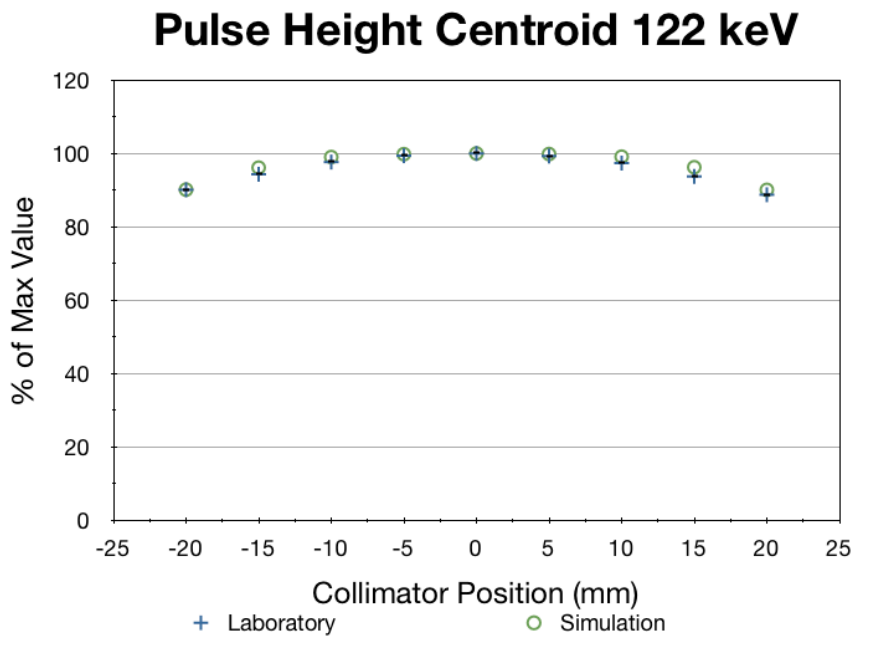

Figure 6: Comparison of the pulse height centroid in the laboratory detector and the simulated detector. An $122 \mathrm{keV}$ incident gamma ray was used to simulated the ${ }^{57} \mathrm{Co}$ source used in the laboratory.
The simulated MAPMT approximates the true MAPMT design with a $49 \mathrm{~mm} \times 49 \mathrm{~mm} \times 1.5 \mathrm{~mm}$ quartz face and an array of $6 \mathrm{~mm} \times 6 \mathrm{~mm}$ blocks to represent each anode. The block material is defined to have absorption properties equal to the quantum efficiency of the photocathode in the Hamamatsu H8500.

The source is a point source housed in a $500 \mu \mathrm{m}$ tungsten collimator having the same dimensions as the lab collimator. A pencil beam was not used to allow for radiation that might leak through the collimator and for simulating fluorescence photons from the tungsten. Simulated photons from the source covered a full $2 \pi$ steradians in the direction of the detector. The source is defined as a mono-energetic source of either 122 or $60 \mathrm{keV}$ gamma rays.

\section{RESULTS}

In order to optimize the detector for spatial and energy resolution, the simulation first needed to be validated. To this end, the simulations have been compared to data taken in the laboratory with the prototype detector using a ${ }^{57}$ Co source. Using a $500 \mu \mathrm{m}$ tungsten collimator, a scan across the center of the detector face at $5 \mathrm{~mm}$ steps was performed. The data was pedestal subtracted, normalized, and background subtracted before the total signal and light distribution were calculated. For the simulations, the all surfaces were considered to be ground with a polish value of either 0 or 1 . The top and side surfaces have a polish value of 0 while the bottom surface has a polish value of 1 .

For $122 \mathrm{keV}$ photons, the lab data showed an edge event produced $\sim 90 \%$ of the signal of a center event. The energy resolution of the experimental data was calculated to be $\sim 10.8 \%$. To match the experimental energy resolution pulse height centroid, the reflectivity of the simulated scintillator's lateral sides was set to 0.6 and the top was set to 0.95 . Figure 6 shows the pulse height centroid as a function of position for the simulated data and the experimental data. This The energy resolution found by the simulations was $\sim 10.0 \%$.
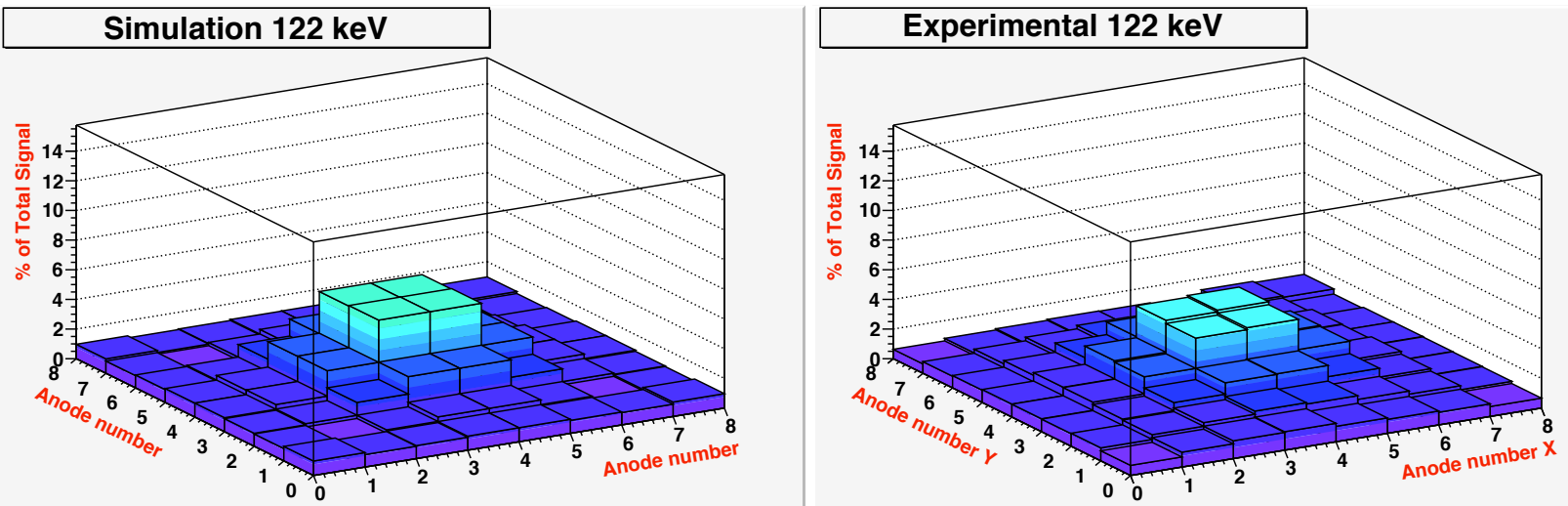

Figure 7: Simulated light distribution from an $122 \mathrm{keV}$ incident gamma ray (left) and the light distribution from a ${ }^{57} \mathrm{Co}$ source (right). Each anode is shown as the average percent of the total signal from a single event. 
The light distribution for the laboratory and simulation data were also compared. A two dimensional plot was made showing the percent of the total signal for each anode, see Figure 7. These plots show that using the reflectivity settings determined by comparing the energy resolution and pulse heigh centroid, the simulation produces a light distribution with similar features to that of the prototype detector.

\section{CONCLUSION}

A Monte Carlo simulation was created using the Geant 4 toolkit to facilitate the optimization the proposed CASTER gamma ray detector. The simulation results have been compared to experimental data taken with a prototype detector and a ${ }^{57}$ Co source. The pulse height centroid and the energy resolution of these data has helped determine some of the initial simulation variables. To accurately model the proposed detector further testing and refinement of variables in the simulation is needed. Comparison to other gamma ray sources, for example ${ }^{241} \mathrm{Am}$, will also be used to help validate the simulation.

When the simulation has been validated, changes to the detector design to optimized the position and energy resolution can be tested. These changes will include scintillator thickness, type, and surface treatments. The type of PMT photocathode and number of anodes will also be tested.

The first test of the detector outside the laboratory will be as a secondary experiment on a high altitude balloon flight. Testing and development of the signal readout equipment for this experiment is being preformed parallel to the detector optimization. The signals from the MAPMT will be collected using an Application Specific Integrated Circuit (ASIC). The balloon flight is set to take place in the fall of 2011.

\section{REFERENCES}

[1] White, N., "Beyond Einstein: scientific goals and missions," Adv. Space Res., 35, 96-105 (2005).

[2] McConnell, M. L. et al., "The CASTER black hole finder probe," New Astronomy Reviews, 50, 633-636 (2006).

[3] Anger, H. O., "Scintillation Camera," Rev. Sci. Instrum., 29, 1958 (1958).

[4] McConnell, M. L. et al., "CASTER: a concept for a Black Hole Finder Probe based on the use of new scintillator technologies," UV, X-Ray, and Gamma-Ray Space Instrumentation for Astronomy XIV, ser. Proc. SPIE, O. H. W. Siegmund, Ed., 5898, 1 (2005).

[5] van Loef, E. V. D. et al., "High Energy Resolution Scintillator: Ce ${ }^{3+}$ Activated $\mathrm{LaBr}_{3}$," Appl. Phys. Lett., 79, 1573-1575 (2001).

[6] S. Agostinelli et al., "GEANT4 - a simulation tool kit," Nucl. Instrum. Meth., A 506, 250-303 (2003).

[7] Saint Gobain 2006, BrillianceTM380 Technical data sheet, available at: www.detectors.saint-gobain.com.

[8] Hamamatsu Photonics, H8500 PSPMT data sheet, Japan, available at: www.hamamatsu.com.

[9] Lo Meo, S. et al., "A Geant4 simulation code for simulating optical photons in SPECT scintillation detectors," J. Intrum., 4, 7002 (2009).

[10]A. Levin and C. Moisan, "A more physical approach to model the surface treatment of scintillation counters and its implementation into DETECT,” IEEE Nucl. Sci. Symp. Conf. Rec., 2, $702-706$ (1996).

[11] Janecek, M., Moses, W., "Simulating Scintillator Light Collection Using Measured Optical Reflectance," IEEE Trans. on Nucl. Sci., 57(3), 964-970 (2010). 